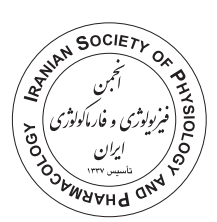

\title{
Pretreatment with p-coumaric acid protect rat's liver against ischemia-reperfusion injury
}

\author{
Razieh Kazemzadeh ${ }^{1}$, Layasadat Khorsandi², Maryam Radan ${ }^{3}$, Samireh Ghafouri ${ }^{3}$, Seyyed Ali Mard ${ }^{1 *}$ (ID)
}

\begin{abstract}
1.Persian Gulf's Physiology Research Center, Medical Basic Sciences Research Institute, Alimentary Tract Research Center, Clinical Sciences Research Institute, Department of Physiology, School of Medicine, Ahvaz Jundishapur University of Medical Sciences, Ahvaz, Iran

2. Cellular and Molecular Research Center, Department of Anatomical Sciences, Medical Basic Sciences Research Institute, School of Medicine, Ahvaz Jundishapur University of Medical Sciences, Iran

3. Persian Gulf's Physiology Research Center, Medical Basic Sciences Research Institute, Department of Physiology, School of Medicine, Ahvaz Jundishapur University of Medical Sciences, Ahvaz, Iran
\end{abstract}

\section{ABSTRACT}

Introduction: Many physiological, biochemical and toxicological reactions are occurred in liver. Therefore, healthy function of this organ is vital for the whole body. In spite of having potent endogenous antioxidant system, lots of reactions in liver make it more susceptible to stressors. It is established that improving the potency of liver antioxidants can increase its ability to resist against different kinds of oxidative stressors. Therefore, this study designed to determine whether p-coumaric alleviate ischemia-reperfusion-induced hepatic injury (IRI) in rats.

Methods: Thirty-two rats were randomly assigned in sham, p-coumaric acid (PC), ischemia-reperfusion (IR) and p-coumaric acid pretreated IR (PC+IR) groups $(n=8$ in each group). Animals in sham group underwent laparotomy but not IR injury; rats in PC group did not experience any surgical procedures; IR and PC+IR groups underwent hepatic IR injury. $\mathrm{P}$-coumaric acid at $100 \mathrm{mg} / \mathrm{kg}$ were given for 7 consecutive days to $\mathrm{PC}$ and $\mathrm{PC}+\mathrm{IR}$ groups. The last dose of p-coumaric acid was injected just before surgery on 7 th days of experiment. The levels of malondialdehyde, TAC, ALT and AST were determined. A molecular evaluation to quantify the gene expression of SOD and GPx was done in liver homogenate.

Results: P-coumaric mitigated the hepatic injuries induced by IR and improved TAC, ALT, AST, SOD and GPx. This pretreatment was also decreased MDA level.

Conclusion: The current outcomes showed that PC via improving the endogenous level of antioxidants in liver tissues and inhibiting IR-induced inflammation maintain the liver structure and function of liver against IR.

\section{Introduction}

Ischemia-reperfusion (IR) injury results from the blood supply failure following blood vessel closure during liver transplantation and it can be one of the main cause of graft failure. The pathophysiological

Keywords:
p-coumaric acid
GPX
SOD
TAC
Rat

Keywords:

GPX

Rat

\footnotetext{
* Corresponding author: Seyyed Ali Mard, mard-sa@ajums.ac.ir

Received 11 March 2020; Revised from 1 August 2020; Accepted 4 August 2020

Citation: kazemzadeh R, Khorsandi L, Radan M, Ghafouri S, Mard SA. Pretreatment with p-coumaric acid protect rat >s liver against ischemia-reperfusion injury. Physiology and Pharmacology 2021; 25: 69-75. http://dx.doi.org/10.32598/ppj.25.1.20
}

mechanisms of hepatic IR include a variety of oxidative stress responses. Numerous studies have also showIntroduction Ischemia-reperfusion (IR) injury results from the blood supply failure following blood vessel closure during liver transplantation and it can be one of 
the main cause of graft failure. The pathophysiological mechanisms of hepatic IR include a variety of oxidative stress responses. Numerous studies have also shown that reactive oxygen species are important mediators in IR-induced injury (Li et al., 2019). In patient with endstage liver diseases, liver transplantation is an efficient and curative treatment. However, ischemia-reperfusion injury is still a major unsolved problem negatively impacts liver transplantation. Liver injury caused by IR leads to up to $10 \%$ failure of early graft function and also increases the risk of organ rejection and liver dysfunction (Kadono et al., 2017). Induced oxidative stress by reperfusion in the gastrointestinal tract leads to excessive reduced glutathione. In other words, reperfusion increases oxidized glutathione formation. (Schoenberg et al., 1985; Younes et al., 1987). Nilsson and colleagues (1989) showed that an explosive increase in the production of oxidants occurs 2-5 min after reperfusion.

Under normal conditions, the oxygen molecules in the mitochondria are converted to water molecules by the cytochrome oxidase system during four stages of reduction. Usually $1-2 \%$ of these molecules escape the cytochrome system and produce free radicals that are neutralized by the endogenous antioxidant defense system of superoxide dismutase (SOD) and glutathione peroxidase (GPx) enzymes (Cerqueira et al., 2005). The activity of antioxidant enzymes SOD and GPx is higher in the liver than in other tissues. Oxygenated water is converted to water and $\mathrm{O} 2$ by the catalase enzyme. GPx is a selenoprotein that reduces lipid and non-lipid hydroperoxides such as $\mathrm{H} 2 \mathrm{O} 2$ when oxidized to glutathione. Liver IR disrupts and reduces the activity of these antioxidants (Yuan et al., 2005).

Malondialdehyde (MDA) is a product of lipid peroxidation produced by free radicals. SOD enzymes are antioxidant enzymes that are involved in detoxification of hydrogen superoxide anion. Increased MDA and decreased SOD activity can be considered as indicators of disrupting the balance between oxidant and antioxidant substances resulting in oxidative stress (Kiris et al., 2007; Paller et al., 1984). Alanine amino transferase (ALT) and aspartate amino transferase (AST) are liver functional enzymes used to diagnose liver diseases and injuries. Studies have shown that the plasma levels of these enzymes increase following liver diseases (Carson et al., 2015; Popova and Buravkova, 2006).

Polyphenols, which exist naturally in nutrients of plant origin, are used as preservatives in the food industry because of their particular antioxidants and anti-inflammatory properties. Among the phenolic acid derivatives, a subgroup of polyphenols, p-coumaric acid (PC) is wellknown compound with these properties. PC is found in edible plants such as groundnuts, tomatoes, carrots, sage and garlic, as well as in beverages such as wine, vinegar, tea, coffee and beer (Mitani et al., 2018). Tyrosine ammonia-lyase converts tyrosine into PC in shikimic acid pathway. P-coumaric acid has been indicated that possesses potent antioxidant properties including scavenging free radicals and up-regulating of endogenous antioxidant enzymes. Furthermore, studies have found that PC could decrease apoptosis proteins and increase nuclear respiratory factors 1 , which it in turn could improve mitochondrial function in stress condition (Urfalioğlu et al., 2017)

Since, oxidative stress is an inevitable events can be occurred under many stressful situations, preventing/ alleviating its effect by different and efficient strategies such as promoting the endogenous antioxidants by administration antioxidant compounds can be helpful. Therefore, this study designed to investigate the preventive effect of p-coumaric acid as an antioxidant in mitigating/preventing the effect of hepatic ischemia-reperfusion injury in rats.

\section{Material and methods}

\section{Animals}

Thirty-two male Wistar rats $(150-200 \mathrm{~g})$ were used in this study. The animals purchased from the animal center of AJUMS, Ahvaz, Iran. They received a standard diet chow and had free access to tap water. They kept in standard condition with room temperature (20$25^{\circ} \mathrm{C}$ ) and 12/12 hours light-dark cycle. All protocols were performed in accordance with ethics committee of Ahvaz Jundishapur University of Medical Sciences (IR. AJUMS.ABHC.REC.1398.050).

\section{Grouping and surgical procedures}

Four groups of male Wistar rats were randomly assigned in sham, p-coumaric acid (PC), ischemia-reperfusion (IR) and p-coumaric acid pretreated IR (PC+IR) groups ( $n=8$ in each group). Animals in sham group underwent laparotomy but not IR injury; rats in PC group did not experience any surgical procedures; IR and PC+IR groups underwent hepatic IR injury. 


\section{Medications}

All groups except PC+IR rats were received vehicle (7\% DMSO diluted with $\mathrm{N} / \mathrm{S} ; 2 \mathrm{ml} / \mathrm{kg}$ ) intraperitoneally (Mard et al., 2016) for a week on a daily basis. Animals in PC+IR and PC groups received p-coumaric acid at $100 \mathrm{mg} / \mathrm{kg}$ (Sigma-Aldrich) for seven consecutive days. The last dose of p-coumaric acid was injected just before surgery on the 7th days of experiment. Rats in sham, IR and PC+IR groups underwent anesthesia with a single injection of anesthetics $(60 \mathrm{mg} / \mathrm{kg}$ ketamine $+15 \mathrm{mg} / \mathrm{kg}$ xylazine; Alfasan Co.Woerden-Holland) on the seventh day of experiment. Then a midline laparotomy were done. IR and PC+IR groups were also experienced ischemia-reperfusion injury as mentioned in detail below.

\section{Surgical procedures}

By using a clamp artery, a partial ischemia (70\%) for $45 \mathrm{~min}$ was performed and to allow reperfusion, the clamp removed. The reperfusion period was $60 \mathrm{~min}$. After that rats were sacrificed by cardiac puncture, their livers removed and washed with physiologic saline. Then, two tissue samples were resected. A sample fixed in formalin $10 \%$ solution for histopathology study and another tissue sample was snap-frozen in liquid nitrogen and kept at $-80^{\circ} \mathrm{C}$ until determination of liver enzymes.

\section{Determination of hepatic aminotransferases}

To measure hepatic aminotransferases, a blood samples were taken, centrifuged at $3000 \mathrm{rpm}$ for $10 \mathrm{~min}$ and then the serums stored at $-20^{\circ} \mathrm{C}$ until evaluation. The levels of ALT and AST were determined using commercial kits according to the manufacturer's protocol (Pars Azmoon Company, Iran) by a serum autoanalyzer (BT1500-A-A, Rome, Italy).

\section{Quantifying the gene expression of antioxidants}

To quantify the mRNA expression of the targeted antioxidants, total RNAs were extracted from the frozen tissue using RNeasy plus mini kit according to manufacturer's instruction. After that the concentration and purity of extracted RNAs were determined using a nanodrop at wavelengths 260 and $280 \mathrm{~nm}$ (NanodropThermo Scientific S.N: D015). Then, $1 \mu \mathrm{g}$ of RNA was used to synthesize cDNA using Qiagen kit (Quantitect Reverse Transcription Kit) in accordance with the manufacturess instruction. Finally, the expression of SOD,
GPx and housekeeping gene (GAPDH) were determined by semi-quantitative real-time PCR (qRT-PCR) using Roche Diagnostics $96^{\mathrm{R}}$. The used primers were as follows: SOD (F: CGGGACTACACCGAAATGAA and R: GAGCCTTCTCACCATTCACC), GPx (F: CGGGACTACACCGAAATGAA and R:GAGCCTTCTCACCATTCACC) and GAPDH (F: TGCTGGTGCTGAGTATGTCGTG and R: CGGAGATGATGACCCTTTTGG). The final volume of PCR reactions were $20 \mu \mathrm{l}$. PCR reactions were done with the following temperatures and timing : incubation at $95^{\circ} \mathrm{C}$ for $15 \mathrm{~min}$ to activate DNA Taq polymerase, followed by 40 cycles at $95^{\circ} \mathrm{C}$ for $20 \mathrm{~s}, 51^{\circ} \mathrm{C}$ for $30 \mathrm{~s}$ and $72^{\circ} \mathrm{C}$ for $30 \mathrm{~s}$. Moreover, the no-template negative control $(\mathrm{H} 2 \mathrm{O})$ was routinely run in every single PCR. The gene expression of housekeeping mRNA- GAPDH- was used to normalize the level of gene expression. The comparative cycle of threshold $(\mathrm{Ct})$ method was used for determining the relative quantification of gene expression, using the arithmetic formula $\left(2^{-\mathrm{AACt}}\right)$.

\section{Determination of MDA and TAC}

To measure the level of MDA and total antioxidant capacity (TAC), frozen liver was homogenate at proper volume PBS $(1 \mathrm{ml}, 50 \mathrm{mM}, \mathrm{pH} 7.4)$ using a homogenizer (Sigma; D-37520 Osterode am Harz; Germany) and then centrifuged for $15 \mathrm{~min}$ at $15000 \mathrm{rpm}$. Commercial kits were used to determine the levels of MDA and TAC in the liver homogenates (Zellbio, Germany).

\section{Statistical analysis}

The data are presented as mean \pm SEM. To analyze the data, one-way analysis of variance (ANOVA) followed by Tukey>s test was used. A probability less than 0.05 considered as statistically significant.

\section{Results}

Effect of hepatic IR injury and p-coumaric acid pretreatment on hepatic aminotransferases

As shown in Table 1, the serum levels of ALT and AST significantly increased following hepatic IR injury $(P<0.001)$. These levels in PC+IR rats improved by pretreatment with $\mathrm{p}$-coumaric acid.

Effect of hepatic IR injury and p-coumaric acidpretreatment on total antioxidant capacity and lipid peroxidation 
TABLE 1: Effect of hepatic IR injury and p-coumaric acid pretreatment on liver functional tests. ${ }^{* *} P<0.01$ significant increase compared to sham, PC and PC-IR groups. Data are expressed as mean $\pm \mathrm{SEM}$.

\begin{tabular}{|ccc|}
\hline Group & AST $(\mathbf{U} \backslash \mathbf{L})$ & ALT $(\mathbf{U} \backslash \mathbf{L})$ \\
\hline IR & $5.348 \pm 00.12^{* *}$ & $5.455 \pm 0.05^{* *}$ \\
\hline Sham & $4.7 \pm 0.03$ & $4.485 \pm 0.04$ \\
\hline PC & $4.5 \pm 0.04$ & $4.003 \pm 0.04$ \\
\hline PC+IR & $4.89 \pm 0.03$ & $4.817 \pm 0.08$ \\
\hline
\end{tabular}

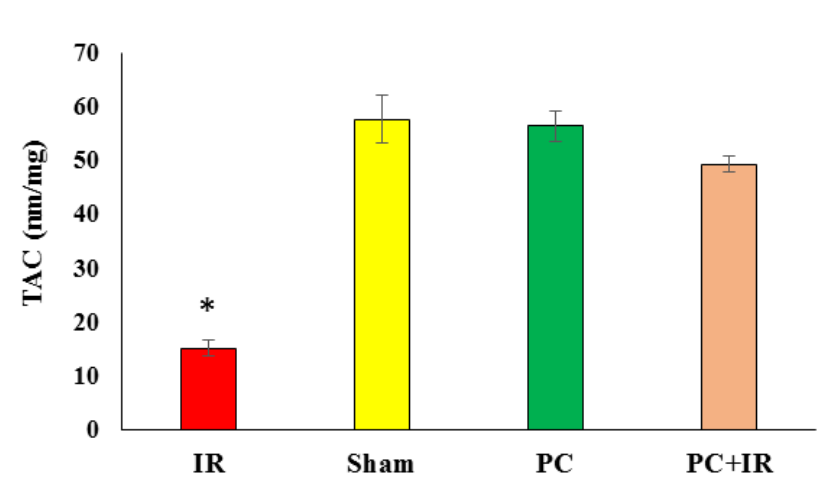

FIGURE 1. Effect of hepatic IR injury and p-coumaric acid pretreatment on TAC level in rat's liver. Hepatic IR injury decreased liver level of TAC while pretreatment with p-coumaric acid reverted this level to normal. Sham, p-coumaric acid (PC), ischemia-reperfusion (IR) and p-coumaric acid pretreated IR (PC+IR) groups $(n=8$ in each group). " $P<0.05$ versus sham, $\mathrm{PC}$ and $\mathrm{PC}+\mathrm{IR}$ groups. Data are expressed as mean \pm SEM.

TAC decreased following hepatic IR injury (Figure. 1). Pretreatment with p-coumaric acid reverted the level of this variable to normal. As demonstrated in Figure 2 , the level of MDA as a criterion for lipid peroxidation significantly increased after hepatic IR injury. Seven days pretreatment with p-coumaric acid decreased MDA level to near normal.

Effect of hepatic IR injury and p-coumaric acid pretreatment on gene expression of GPX

As demonstrated in Figure 3, hepatic IR injury significantly decreased mRNA level of GPx in ratss liver compared to sham group $(P<0.01)$. Pretreatment with p-coumaric acid reverted this level in IR rats to normal. This level in PC+IR rats was as much as in sham group. The level of GPx mRNA expression was the highest among the experimental groups.

Effect of hepatic IR injury and p-coumaric acid pretreatment on gene expression of $S O D$

Figure 4 shows that hepatic IR injury significantly decreased mRNA level of SOD in rat ss liver compared

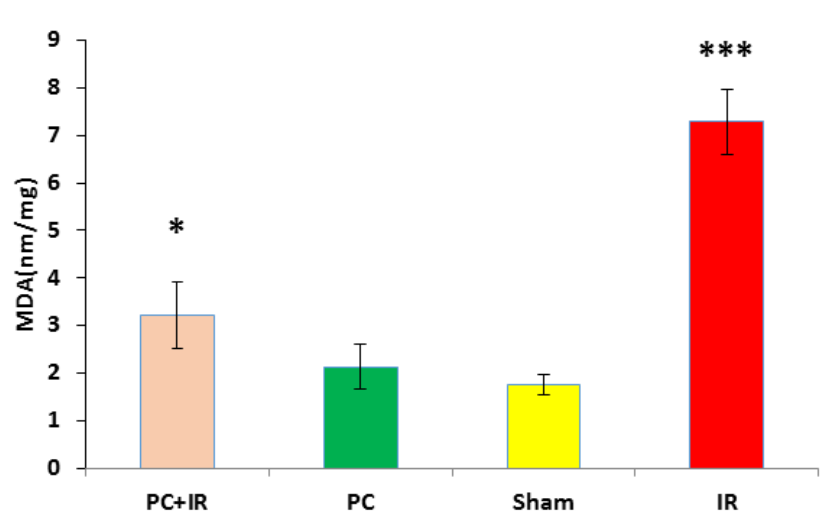

FIGURE 2. Effect of hepatic IR injury and p-coumaric acid pretreatment on MDA level in rat's liver. The hepatic level of MDA increased after liver IR injury while pretreatment with p-coumaric acid significantly improved this level. Sham, p-coumaric acid (PC), ischemia-reperfusion (IR) and p-coumaric acid pretreated IR (PC+IR) groups ( $\mathrm{n}=8$ in each group). ${ }^{* * *} P<0.001$ versus sham and $\mathrm{PC}$ groups. ${ }^{*} P<0.05$ versus sham group. Data are expressed as mean \pm SEM.

to sham group $(P<0.05)$. Pretreatment with $\mathrm{p}$-coumaric acid reverted this level in IR rats to normal. The level of SOD mRNA expression in PC group was non-significantly higher than in sham rats.

\section{Discussion}

This study showed that partial liver ischemia (45min) followed by one hour reperfusion increased the serum levels of AST and ALT. Hepatic IR injury was also increased the liver level MDA while decreased TAC level and mRNA expression of GPx and SOD in liver tissue. P-coumaric acid pretreatment effectively protected liver function against liver I/R injury through decreasing liver MDA level and improving serum levels of AST and ALT. The antioxidant potency of liver tissue increased by p-coumaric acid as evidenced by increasing TAC and mRNA expression of SOD, GSH and CAT in liver tissue (Ekinci Akdemir et al., 2017).

Liver amino transaminases (AST and ALT) release from hepatocytes to systemic circulation and elevate in serum during and after structural damages, necrosis and cellular leakage ( Faqi, 2016). The present findings 


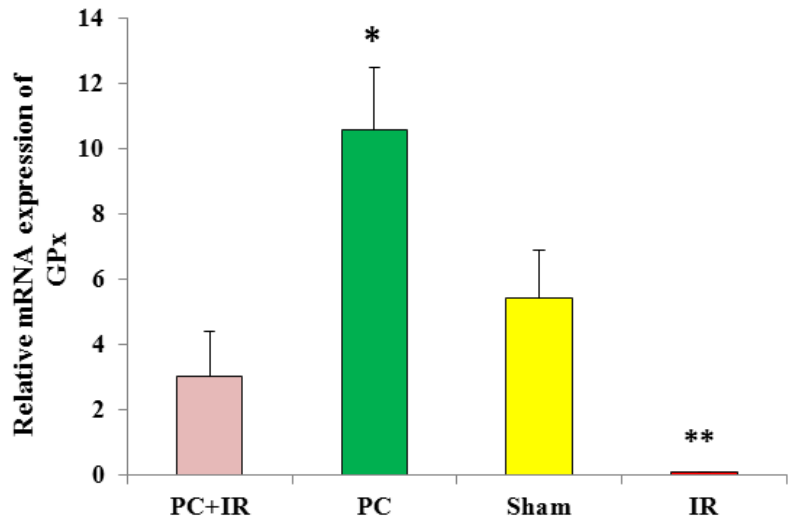

FIGURE 3. The effect of ischemia-reperfusion injury and p-coumaric acid pretreatment on mRNA level of GPx in rat's liver. The mRNA level of GPx in liver decreased following ischemia-reperfusion injury. Pretreatment with p-coumaric acid significantly prevented this decrease. Sham, p-coumaric acid (PC), ischemia-reperfusion (IR) and $\mathrm{p}$-coumaric acid pretreated IR (PC+IR) groups $(\mathrm{n}=8$ in each group). ${ }^{*} P<0.05$ versus sham group. ${ }^{* *} P<0.01$ versus sham group

showed that liver IR increased serum AST and ALT, while p-coumaric acid pretreatment at $100 \mathrm{mg} / \mathrm{kg}$ for seven consecutive days before inducing hepatic IR injury decreased these levels. Previous studies have been shown that IR injury through increasing reactive oxygen species production and lipid peroxidation, disrupt cell membrane permeability and integrity (Klune and Tsung, 2010). Another study has been shown antioxidant compounds maintain cell membrane integrity through inhibiting lipid peroxidation and consequently improving AST and ALT serum levels (Akbari et al., 2017). Therefore, the above mentioned reports along with the present results concluded that $\mathrm{p}$-coumaric acid as an antioxidant with maintaining cell membrane integrity decreased AST and ALT release and improved these levels.

Our finding showed liver MDA level increased following partial liver IR. However, the increased level of MDA (as a marker of lipid peroxidation) related to liver IR could represent the side effects of oxidative stress. The prior studies have reported that liver IR induce oxidative stress and increase MDA level (Tavafi et al., 2020). These findings concluded that liver IR induce liver oxidative stress, increase lipid peroxidation, damage hepatocyte cell, increase cell membranes permeability that leads to an increment in the serum levels of ALT and AST. This study showed that p-coumaric acid pretreatment decreased liver MDA level. A previous report has been indicated p-coumaric acid pretreatment decreased MDA level in cerebral IR injury (Sakamula and Thongasa, 2018). Therefore, these results together show that one of the involved mechanism by which p-coumaric

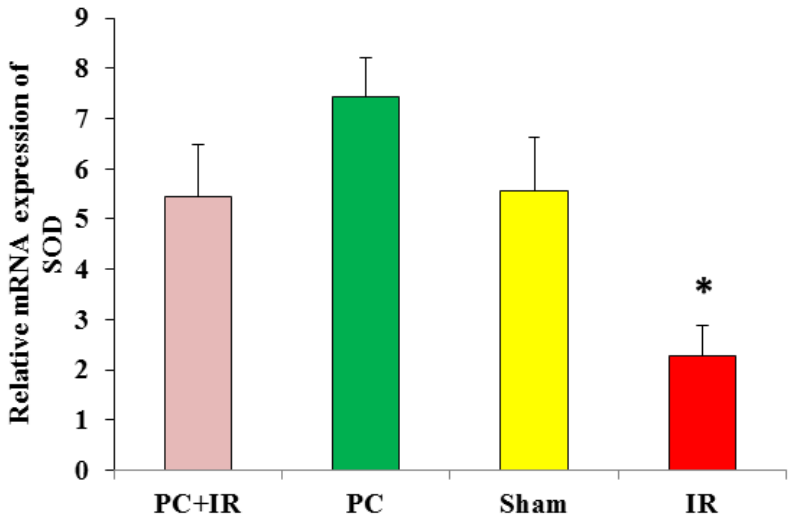

FIGURE 4. Effect of hepatic IR injury and p-coumaric acid pretreatment on SOD expression level in rat's liver. Hepatic IR injury decreased mRNA level of SOD in liver while pretreatment with p-coumaric acid mitigated this increase. Sham, p-coumaric acid (PC), ischemia-reperfusion (IR) and p-coumaric acid pretreated IR (PC+IR) groups ( $\mathrm{n}=8$ in each group). ${ }^{*} P<0.05$ versus sham group. Data are expressed as mean \pm SEM.

acid exerts its tissue protection effect is its antioxidant property.

Our finding showed that liver IR decreased liver TAC level significantly while p-coumaric acid pretreatment improved this level. Liver IR increased oxidative stress, disturb oxidant-antioxidant balance and oxidant mediators conquered antioxidant defense. Previous studies have been shown that liver IR reduce liver antioxidant levels (Altay et al., 2016). Another study also showed that p-coumaric acid improve antioxidant capacity and exhibit protective effect on oxidative stress (Sakamula and Thong-asa, 2018). Our finding was in consistent with prior studies shown that liver IR decreased antioxidant potency while p-coumaric acid pretreatment increased TAC and improved antioxidant capacity.

The current result showed that, liver ischemia-reperfusion decreased mRNA levels of SOD and GPx in liver tissue, while p-coumaric acid pretreatment increased these levels to sham group. A pervious report has been shown that phenolic components (gallic acid, ferulic acid and p-coumaric acid) at $100 \mathrm{mg} / \mathrm{kg}$ increased the cardiac SOD, GPx and CAT mRNA levels (Yeh et al., 2009). Therefore, these findings suggest that $\mathrm{p}$-coumaric acid not only increased and potentiated the endogenous potency of antioxidants at the non-genomic levels but it also increased this ability at the genomic levels by up-regulating the transcription of antioxidants mRNAs.

\section{Conclusion}

In conclusion, the present findings showed that p-coumaric acid protect liver function by improving liver 
enzymes. The probable involved mechanism by which p-coumaric exert its beneficial effect against IR injury was improving endogenous antioxidants potency.

\section{Acknowledgment}

This paper was extracted from the M.Sc desertification of Miss Razieh Kazemzadeh (M.Sc student in medical physiology) of the Ahvaz Jundishapur University of Medical Sciences. The authors gratefully acknowledge the help and financial support of Physiology Research Center of Ahvaz Jundishapur University of Medical Sciences (grant No. APRC-9810), Ahvaz, Iran.

\section{Conflict of interest}

All authors declare that they have no any conflict of interest.

\section{References}

Akbari G, Mard SA, Dianat M, Mansouri E. The hepatoprotective and MicroRNAs downregulatory effects of crocin following hepatic ischemia-reperfusion injury in rats. Oxid Med Cell Longev 2017; 2017: 1702967. https://doi. org/10.1155/2017/1702967

Altay N, Karahan MA, Büyükfirat E, Yeşilay A, Yüce HH, Aydoğan H, et al. A combination of dexmedetomidine and thymoquinone is better able to prevent ischemia reperfusion injuries in the liver: an experimental study in rat model. Int J Clin Exp Med 2016; 9: 2521-2527.

Carson P, Hong CJ, Otero-Vinas M, Arsenault EF, Falanga V. Liver enzymes and lipid levels in patients with lipodermatosclerosis and venous ulcers treated with a prototypic anabolic steroid (stanozolol) a prospective, randomized, double-blinded, placebo-controlled trial. Int J Low Extrem Wounds 2015; 14: 11-8. https://doi. org/10.1177/1534734614562276

Cerqueira NF, Hussni CA, Yoshida WB. Pathophysiology of mesenteric ischemia/reperfusion: a review. Acta Cirurgica Brasileira 2005; 20: 336-43. https://doi.org/10.1590/ S0102-86502005000400013

Ekinci Akdemir FN, Albayrak M, Çalik M, Bayir Y, Gülçin. The protective effects of $\mathrm{p}$-coumaric acid on acute liver and kidney damages induced by cisplatin. Biomedicines 2017; 5: 18. https://doi.org/10.3390/biomedicines5020018

Faqi AS, editor. Biomarkers in nonclinical drug development. In: A comprehensive guide to toxicology in nonclinical drug development. 2nd Edition. Boston: Academic Press, 2016.
Kadono K, Uchida Y, Hirao H, Miyauchi T, Watanabe T, Iida $\mathrm{T}$, et al. Thrombomodulin attenuates inflammatory damage due to liver ischemia and reperfusion injury in mice in tolllike receptor 4-dependent manner. Am J Transplant 2017; 17: 69-80. https://doi.org/10.1111/ajt.13991

Kiris I, Okutan H, Savas C, Yonden Z, Delibas N. Gadolinium chloride attenuates aortic occlusion-reperfusion-induced myocardial injury in rats. Saudi Med J 2007; 28: 347.

Klune JR, Tsung A. Molecular biology of liver ischemia/ reperfusion injury: established mechanisms and recent advancements. Surg Clin North Am 2010; 90: 665-77. https:// doi.org/10.1016/j.suc.2010.04.003

Li S, Fujino M, Takahara T, Li XK. Protective role of heme oxygenase-1 in fatty liver ischemia-reperfusion injury. Med Mol Morphol 2019; 52: 61-72. https://doi.org/10.1007/ s00795-018-0205-Z

Mard SA, Pipelzadeh MH, Teimoori A, Neisi N, Mojahedin S, Khani MZ, et al. Protective activity of crocin against indomethacin-induced gastric lesions in rats. J Nat Med 2016; 70: 62-74. https://doi.org/10.1007/s11418-015-0938-0

Mitani T, Mimura H, Ikeda K, Nishide M, Yamaguchi M, Koyama H, et al. Process for the purification of cis-p-coumaric acid by cellulose column chromatography after the treatment of the trans isomer with ultraviolet irradiation. Anal Sci 2018; 34: 1195-9. https://doi.org/10.2116/analsci.18P102

Nilsson U, Lundgren O, Haglind E, Bylund-Fellenius A. Radical production during in vivo intestinal ischemia and reperfusion in the cat. Am J Physiol Gastrointest 1989; 257: G409-14. https://doi.org/10.1152/ajpgi.1989.257.3.G409

Paller MS, Hoidal J, Ferris TF. Oxygen free radicals in ischemic acute renal failure in the rat. J Clin Investig 1984; 74 : 1156-64. https://doi.org/10.1172/JCIcvv111524

Popova J, Buravkova L. Blood biochemical parameters in women during long-term simulated hyperoxic diving up to 8 ATA. Undersea Hyperb Med 2006; 33: 211-6.

Sakamula R, Thong-asa W. Neuroprotective effect of p-coumaric acid in mice with cerebral ischemia reperfusion injuries. Metab Brain Dis 2018; 33: 765-73. https://oi. org/10.1007/s11011-018-0185-7

Schoenberg MH, Fredholm BB, Haglund U, Jung H, Sellin D, Younes M, et al. Studies on the oxygen radical mechanism involved in the small intestinal reperfusion damage. Acta Physiol Scand 1985; 124: 581-9. https://doi. org/10.1111/j.1748-1716.1985.tb00051.x

Tavafi M, Ahmadvand H, Tamjidipour A, Hasanvand A. Rosmarinic acid ameliorates renal ischemia reperfusion dam- 
age in rats. J Nephropharmacol 2020; 9: e15. https://doi. org/10.34172/npj.2020.15

Urfalioğlu A, Yazar FM, Bilal B, Tolun Fİ, Öksüz H, Boran ÖF, et al. The effect of p-coumaric acid and ellagic acid on the liver and lungs in a rat model of sepsis. Asian Biomed 2017; 11: 217-25.

Yeh CT, Ching LC, Yen GC. Inducing gene expression of cardiac antioxidant enzymes by dietary phenolic acids in rats. $\mathrm{J}$ Nutr Biochem 2009; 20: 163-71. https://doi.org/10.1016/j. jnutbio.2008.01.005
Younes M, Mohr A, Schoenberg M, Schildberg F. Inhibition of lipid peroxidation by superoxide dismutase following regional intestinal ischemia and reperfusion. Res Exp Med 1987; 187: 9-17. https://doi.org/10.1007/BF01854963

Yuan GJ, Ma JC, Gong ZJ, Sun XM, Zheng SH, Li X. Modulation of liver oxidant-antioxidant system by ischemic preconditioning during ischemia/reperfusion injury in rats. World J Gastroenterol 2005; 11: 1825. https://doi. org/10.3748/wjg.v11.i12.1825 